\title{
El reto de la eficiencia energética en Europa y el riesgo del efecto rebote
}

\section{The Challengue of Energy Efficiency in Europe and the Rebound Effect's Risk}

\author{
José Manuel Cansino ${ }^{1}$ \\ Universidad de Sevilla (España) y Universidad \\ Autónoma de Chile (Chile)
}

Recibido: 10-09-20

Aceptado: 29-09-20

\section{Resumen}

Este artículo explora la manera en la que las autoridades de la Unión Europea afrontan el riesgo de un efecto rebote que dificulte la efectividad de los esfuerzos hechos para mejorar la eficiencia energética. La elección de la zona de la UE se debe a su liderazgo internacional en la batalla contra el calentamiento global y el cambio climático. El artículo hace una mención específica a España por ser uno de los estados miembros de la UE más concernidos por estos problemas.

Para hacer esto se realiza una sistemática revisión de la literatura siguiendo las técnicas avanzadas que recomienda el estado del arte. Después de esto, los principales hallazgos del artículo son 1) existe un amplio consenso académico en torno a la importancia del efecto rebote como barrera en la mejora de la eficiencia energética, 2) existe una falta de consenso sobre su tamaño, 3) los textos legales

\footnotetext{
${ }^{1}$ (jmcansino@us.es). Catedrático de Economía Aplicada del departamento de Análisis Económico y Economía Política de la Universidad de Sevilla. Entre sus últimas publicaciones cabe destacar: Burneo, Damian, Cansino Muñoz-Repiso, Jose Manuel, Yñiguez Ovando, Rocío: Environmental and Socioeconomic Impacts of Urban Waste Recycling as Part of Circular Economy. The Case of Cuenca (Ecuador). En: "Sustainability", 2020. Vol. 12. N 8; Cansino Muñoz-Repiso, Jose Manuel, Román Collado, Rocío, Molina, Juan C: Quality of Institutions, Technological Progress, and Pollution Havens in Latin America. An Analysis of the Environmental Kuznets Curve Hypothesis. En: "Sustainability", 2019. Vol. 11. No 13. https://doi.org/10.3390/su11133708; Cansino Muñoz-Repiso, Jose Manuel, Roman Collado, Rocío, Merchán, José: Do Spanish energy efficiency actions trigger JEVON ¿S paradox? En: “Energy", 2019. Vol. 181. No August 2019. Pp. 760-770. https://doi.org/10.1016/j.energy.2019.05.210; Cansino Muñoz-Repiso, Jose Manuel, Moreno, Roberto, Quintana, Daniela, Román Collado, Rocío: Health and Heating in the City of Temuco (Chile). Monetary Savings of Replacing Biomass with PV System in the Residential Sector. En: "Sustainability", 2019. Vol. 11. No 19. https://doi.org/10.3390/ su11195205; Roman Collado, Rocío, Cansino Muñoz-Repiso, Jose Manuel, Botia, Camilo: How far is Colombia from decoupling? Two-level decomposition analysis of energy consumption changes. En: “Energy”, 2018. Vol. 148. No 1 April 2018. Pp. 687-700. https://doi.org/10.1016/j.energy.2018.01.141

ORCID: https://orcid.org/0000-0003-1087-5399.
} 
que abordan los objetivos en materia de eficiencia energética habitualmente ignoran el problema del efecto rebote.

Palabras-clave: Efecto Rebote, Eficiencia energética, Unión Europea, España, Cambio climático, Calentamiento global.

\begin{abstract}
This paper explores how the European Union's -EU- Authorities face the risk of a rebound effect limiting the effectiveness of efforts made energy efficiency oriented. Choice of EU zone is due to her leadership in the international battle against global warning and climate change. Specific mention to Spain as one of its more concerned member states is made.

To do that a systematic literature review was conducted following advanced techniques recommended by the state of art. After this, mayor findings of this paper conclude that 1) a broad academic consensus exist on the relevance of the rebound effect as a barrier in energy efficiency improvements, 2) a lack of consensus exits on its size, 3) legal documents focused on energy efficiency targets use to ignore rebound effect problem.
\end{abstract}

Key-words: Rebound Effect, Energy Efficiency, European Union, Spain, Climate Change, Global Warming.

\title{
1. Introducción
}

La mejora en la eficiencia energética entendida como la reducción de los requerimientos energéticos por unidad de producto es una de las más relevantes políticas sobre las que descansa la transición a un modelo económico neutro en emisiones de gases de efecto invernadero (GEI). Esta mejora puede ser el resultado del progreso tecnológico, de la mejora en la calidad de los materiales y/o de la mejora en la organización de los procesos productivos. Para la Agencia Internacional de la Energía, la mejora en la eficiencia energética a escala global sería la responsable de un tercio de los esfuerzos necesarios para alcanzar en 2040 lo que denominan "escenario de desarrollo sostenible". Este escenario permitiría 1) mantener el aumento de la temperatura del Planeta muy por debajo de los $2^{\circ}$ centígrados con respecto a la etapa preindustrial, 2) el acceso universal a la energía y 3) la reducción sustancial de la contaminación del aire (IEA, 2020). Desde la perspectiva de la gobernanza internacional de la lucha contra el Cambio Climático (CC) y el calentamiento global, el anterior es un objetivo más ambicioso que el asumido por los países firmantes del Acuerdo de París de 2015 (Naciones Unidas, 2015). 
La Unión Europea (UE) y, con especial compromiso alguno de sus países miembros como España, han asumido el reto de mejorar la eficiencia energética tanto en los sectores productivos de sus economías como en el sector residencial. Tanto es así que la eficiencia energética se concibe como acción prioritaria contribuyendo a la aplicación del principio "primero, la eficiencia energética". Se le otorga, por tanto, mejor prelación sobre actuaciones tales como la mayor penetración de las energías renovables en la matriz energética, el cambio en el parque de vehículos reemplazando los movidos por motores de combustión tradicionales por vehículos flexibles o la gestión inteligente de redes de transporte y distribución de electricidad. Estos compromisos no sólo no han sido devaluados como consecuencia de la crisis derivada de la pandemia de la COVID-19, sino que se han insertado en el paquete de estímulos económicos aprobados por el Consejo Europeo el 21 de julio de 2020 (Consejo Europeo, 2020). No obstante, y como era de esperar, la credibilidad de los objetivos establecidos por la UE ha sido respaldada por unos autores (Liobikienè y Butkus, 2017), pero también cuestionada por otros (Weale, 2020).

Aunque existen precedentes anteriores, la actual normativa de referencia en la que se plasma el compromiso de la UE con la mejora de la eficiencia energética es la Directiva 2012/27/UE, recientemente actualizada por la Directiva 2018/2002/UE. La primera Directiva establecía un objetivo de mejora de la eficiencia energética del $20 \%$ para el año 2020. Esto implicaba reducir el consumo de energía final de la UE hasta un nivel no superior a las 1.086 Mtep (millones de toneladas equivalentes de petróleo) y el de energía primaria no por encima de las 1.483 Mtep. La segunda Directiva estableció como objetivo para 2030 una mejora de la eficiencia energética del 32,5\% a escala de la Unión, expresada en consumo de energía primaria o energía final. Las previsiones realizadas en 2007 mostraron un consumo de energía primaria en 2030 de 1.887 Mtep y un consumo de energía final de 1.416 Mtep. Una reducción del $32,5 \%$ correspondería a unos consumos de energía primaria y final de 1.273 Mtep y 956 Mtep en 2030, respectivamente.

El especial compromiso de España en este mismo vector clave en la transición hacia una economía neutra en carbono se ha puesto de manifiesto en el objetivo de reducir el consumo de energía primaria en 2020 de forma que no supere los 123,4 Mtep ni los 87,2 Mtep para la energía final (Ministerio de Industria Energía y Turismo, 2014). Los compromisos quedaron recogidos en el Plan Nacional de Acción de Eficiencia Energética 2017-2020. Actualmente se trabaja en la aprobación del Plan Nacional para el periodo 2021-2030.

Aunque el foco de este artículo se pone en las actuaciones para impulsar la eficiencia energética, éstas no deben contemplarse de manera inconexa respecto a las actuaciones realizadas en otros ámbitos, como el de los modos de transporte y uso de combustibles (Directiva 2014/94/UE), de actuaciones en 
el parque de edificios (Directiva 2010/31/UE) o en la transición a un modelo de desarrollo basado en la economía circular (Comisión Europea, 2015). De hecho, y sobre este último particular la Directiva 2012/27/UE en su versión modificada por la Directiva de 2018, advierte de que los Estados miembros deben prestar una atención particular a las sinergias entre las medidas de eficiencia energética y el uso eficiente de los recursos naturales con arreglo a los principios de la economía circular.

A pesar de la relevancia de las mejoras en la eficiencia energética, los planes de las diferentes autoridades - particularmente de la UE y de Españaparecen no haber incorporado el riesgo de que tales mejoras provoquen un efecto rebote en el uso de la energía. Si ponemos el foco únicamente en la mejora de la eficiencia energética derivada del progreso tecnológico, el efecto rebote aparece cuando, permaneciendo constantes los precios reales de la energía, las ganancias en eficiencia energética aumentan el consumo energético debido a la liberación de fondos que se produce como consecuencia de la disminución inicial de necesidad de recurso energético. Los recursos financieros que quedan liberados estimularían el crecimiento económico y con él un aumento en forma de rebote del consumo energético (Lin y Liu, 2012; Lin et al., 2017; Cansino 2020).

El objetivo de este artículo es triple 1) analizar la literatura disponible orientada a la cuantificación del efecto rebote en la UE para determinar si es o no relevante, 2) analizar cómo la normativa en vigor ha abordado este riesgo y 3) aportar medidas factibles para reducir el riesgo o la magnitud del efecto rebote.

La estructura del artículo es como sigue. Tras la introducción la Sección 2 presenta la metodología y ofrece las principales definiciones relevantes. La Sección 3 muestra el disenso que existe en la medición del ER mientras que la Sección 4 analiza su relevancia para la normativa vigente. Las principales conclusiones se resumen en la Sección 5.

\section{Metodología y definiciones clave}

\subsection{Metodología}

La metodología utilizada consiste en una revisión sistemática de la literatura en la línea de lo recomendado por trabajos previos como los de Tranfield et al., (2003) y Fink (2013). Este método sigue tres pasos para llevar a cabo la revisión sistemática de la literatura 1) Identificar las necesidades de revisión de la literatura de acuerdo con el objetivo del artículo, 2) seleccionar los artículos relacionados que cumplan con un elevado estándar de calidad (esencialmente 
que sean documentos peer review) y 3) presentar las recomendaciones y evidencias identificadas en el proceso de revisión. La revisión se ha realizado principalmente a través de las bases de datos 'Web of Science' (WOS) y Scopus. Para el análisis de la normativa relevante se ha revisado exhaustivamente la base de datos Eur-lex. Finalmente, para la normativa específica de aplicación en España se han rastreado los apartados de legislación de los ministerios con competencias en materia de energía.

\subsection{Definiciones clave}

Formalmente el efecto rebote generado por el progreso tecnológico en un periodo t puede ser definido como uno menos el cociente entre el ahorro real de energía después de la introducción de la medida de mejora tecnológica y el ahorro potencial de energía que podría alcanzarse de no existir efecto rebote y que se deriva de cálculos estrictamente ingenieriles (Arocena et al.; 2016). En definitiva,

$$
E R_{t}=1-\frac{\text { Ahorro real de energía }}{\text { Ahorro potencial de energía }}
$$

Sin embargo, aun siendo un concepto intuitivo, su medición precisa permanece como una cuestión no resuelta limitando así la definición correcta de las actuaciones públicas orientadas a promover eficazmente reducciones en el consumo de energía. Greening et al. (2000) identificaron tres tipos de efecto rebote,

Efecto Rebote Directo (ERD). Este efecto es consecuencia directa de una caída en el precio real de la energía (cantidad de energía requerida por unidad de producto final) como consecuencia de una mejora en la eficiencia energética asociada al progreso tecnológico. La reducción del precio de la energía induciría a consumir más energía.

Efecto Rebote Indirecto (ERI). Junto a lo anterior la caída en el precio real de la energía aumenta la renta disponible de los agentes económicos. Esta renta disponible puede inducir a aumentar la demanda de otros bienes y servicios para cuya producción se requeriría un mayor consumo de energía a través de las cadenas productivas y de las relaciones interindustriales. Dado que el ERD y el IRD se producen simultáneamente aparece la dificultad de medir separadamente uno y otro efecto).

Efecto rebote global (ERG). La reducción en los requerimientos energéticos promovidos por el progreso tecnológico provocará un reajuste en los precios relativos de los bienes y servicios a lo largo de toda la economía. Particularmente los bienes y servicios intensivos en requerimientos energéticos reducirán sus precios absolutos y también relativos respecto al resto de bienes y servicios. Finalmente se producirá un reajuste en la demanda de toda la energía 
para cuyo suministro se puede requerir un aumento en el consumo final de energía. También este efecto es una consecuencia del ERD e igualmente difícil de deslindar (Lin et al., 2017).

\section{Disenso en la relevancia del efecto rebote}

\subsection{Estimaciones globales del ER}

La literatura disponible orientada a la medición del ER coincide en dos hechos; 1) en otorgarle mayoritariamente un papel relevante en el diseño de las políticas energéticas y, específicamente, de eficiencia energética y 2) en el disenso, más que la falta de consenso, sobre la magnitud del mismo. La literatura reciente muestra un inequívoco carácter emergente y ofrece tanto mediciones del ER para amplias zonas económicas (principalmente cuando la metodología usada es la de los modelos de equilibrio general) como para países y regiones específicos. Otra parte notable de la literatura pone el foco tanto en sectores productivos concretos intensivos en el uso de energía como en el sector de los hogares. Una y otra perspectivas -geográfica y sectorial-se analizan a continuación.

Para el conjunto de la economía mundial, Wei y Liu (2017) cifran el ER del consumo de energía en un $70 \%$. Este resultado es principalmente consecuencia del movimiento laboral entre actividades económicas pero también de la sustitución entre consumo de energía y otros factores productivos. La sustitución sería considerable incluso para aquellas actividades en las que el valor de la elasticidad de sustitución entre energía y factores no energéticos es baja. Freire-González (2017) centrándose en la UE-27 ofrece un valor medio del ER ponderado por el PIB de los diferentes países oscilando entre el 73,62 $\%$ y el $81,16 \%$. La metodología empleada por este autor es estática, por lo que no permitiría incorporar el ERG que implicaría reponderar el peso de los sectores productivos intensivos en energía en el conjunto de la actividad económica. El ER subiría al 90 \% según Adetutu et al., (2015, 2016). Junto con las estimaciones del ER para el conjunto de la economía mundial y para la UE-27, Kulmer y Seebauer (2019) lo estiman en el $65 \%$ para la economía austríaca. Por tanto, los cinco resultados anteriores estarían en la misma línea, aunque el ámbito geográfico al que están referidos es significativamente diferente. Importa añadir que el cálculo de Kulmer y Seebauer (2019) lo presentan como valor del ERG al tiempo que cifran el ERD entre el $8 \%$ y el $10 \%$, por lo que la diferencia entre ambos rangos de valores la atribuyen a lo que en el epígrafe anterior hemos definido como ERI. En definitiva, utilizan una definición más estrecha del ER al igualar el ERD a la suma del ERD y 
del ERI cuando, en rigor, están ignorando el efecto rebote que se produce una vez que los sectores productivos más intensivos en energía, después de reducir los precios de sus productos, tendrían una mayor presencia en el conjunto de la actividad económica. A este tercer efecto es al que nosotros identificamos como ERG pero en un sentido diferente al de Kulmer y Seebauer (2019). Una medición apartada de los trabajos citados se encuentra en el interesante artículo de Gillingham et al., (2016), quienes estiman un valor entre el $20 \%$ y el $40 \%$ para el ER cuando éste se define desde una perspectiva microeconómica.

Finalmente, para el caso español Guerra y Sancho (2011) estimaron un ER que varía en un rango del $87,4 \%$ al 90,8\%. Sus resultados estaban en línea con los de Arocena et al. (2016), que encontraron un ER superior al 70\%, pero eran superiores a los hallados por Duarte et al., (2018), que se situaban en el 55,85\% para el año 2030 cuando se consideran mejoras en los electrodomésticos de los hogares y en el transporte. Todos estos resultados están muy por encima del 15\% obtenido por Barker et al. (2007a) para la economía británica en 2007.

Pese a que los cinco resultados anteriores referidos a España no son muy diferentes sería erróneo deducir un notable consenso en la medición del ER inducido por mejoras en la eficiencia energética. Precisamente, sobre esta falta de acuerdo o disenso llaman la atención Vélez-Henao et al. (2019), quienes lo atribuyen, principalmente, al desacuerdo académico sobre la mejor forma de capturar el valor del progreso tecnológico. De hecho, la medición de la contribución del progreso tecnológico al crecimiento económico ha sido siempre una cuestión controvertida. En ausencia de una mejor alternativa, los economistas acostumbramos a aproximar esa contribución a través del denominado Factor de Productividad Total que luego que se formaliza matemáticamente en las habituales funciones de producción Cobb-Douglas (Douglas, 1976; Howart, 1997).

\subsection{Estimaciones del ER en sectores productivos}

La medición separada de los efectos ERD, ERI y ERG no está en absoluto clara. Así, la definición seminal de efecto rebote ofrecida por Herring (2004) parece estar orientada al ERG, sin mención alguna ni al ERD ni al ERI. Pero, retomando el disenso en las mediciones del ER, éste se aprecia fácilmente cuando se desciende desde las mediciones agregadas para el conjunto de la economía a sectores productivos concretos o al sector de los hogares.

Por sectores, uno de los que más atención académica ha recibido es el del transporte, principalmente por carretera, y tanto de mercancías como de pasajeros. En países como España el transporte supone el $40 \%$ del consumo final de energía. Particularmente los vehículos destinados al transporte de mercancías (vehículos comerciales ligeros y camiones) representan el $43 \%$ 
del consumo energético por carretera. El creciente desarrollo del comercio electrónico y la actividad logística que conlleva actúa como un catalizador del uso de transporte de mercancías por carreteras y áreas urbanas pudiendo aumentar más aún su consumo de energía (Ordóñez, 2019).

La posible razón explicativa para haber recibido una atención tan intensa por la literatura estaría en el carácter intuitivo que para este sector tiene el ER; particularmente el ERD. Aunque la literatura ofrece definiciones más elaboradas del ERD, Vivanco et al. (2016) lo ejemplifican recurriendo al símil de que cuando se reduce el consumo de combustible por kilómetro en los automóviles (el precio real del combustible se abarata), los usuarios pueden elegir comprarse automóviles más grandes con un mayor consumo de combustible o aumentar la distancia total de sus viajes.

Llorca y Jamasb (2017) analizaron el ER en el transporte de mercancías para la UE-15 obteniendo un valor del 4\% para el periodo 1992-2012. La magnitud era mayor para aquellos países con combustibles más eficientes y mejor estructura logística. Sin embargo, para Portugal, Matos y Silva (2011) obtuvieron un valor muy superior -el 24,1\%- para los años 1987-2006. Su estudio es de los pocos que analizan mejoras de la eficiencia no sólo desde la perspectiva del progreso tecnológico, sino también en los procesos productivos y en los materiales (concretamente analizando la eficiencia de los combustibles). De hecho, la distinción resulta ser significativa, pues encontraron que los operadores de flotas de vehículos de transporte se mostraban más sensibles a adoptar mejoras en la eficiencia derivadas de cambios en los procesos productivos (diseño de la logística) que en los combustibles.

El transporte de pasajeros por carretera o el uso de turismos por el sector de los hogares también ha recibido notable atención desde el ángulo del efecto rebote. Craglia y Cullen (2020) obtuvieron resultados en línea con los de Llorca y Jamasb (2017) para el transporte de mercancías. Analizando el comportamiento de los usuarios de turismo en el Reino Unido, Craglia y Cullen (2020) encontraron que los vehículos menos eficientes son los que más reaccionan a cambios en los precios implícitos de los combustibles (una mejora en la eficiencia del combustible se asimila a una reducción del precio implícito) y que los conductores de zonas urbanas son más sensibles que los residentes en zonas rurales. En promedio el ER fue del 4,6\%. El disenso académico se pone de nuevo de manifiesto al analizar el trabajo de Frondel et al. (2012) que estudiaron el ER para Alemania durante los años 1997-2009. Para estos autores el ER variaría en un rango entre el 57\% y el 62\%. La magnitud del ER dependería inversamente de la intensidad de la conducción de los miembros del hogar. Los hogares que viajan pocos kilómetros muestran un ER significativamente mayor que los que realizan muchos kilómetros de viaje. Sus resultados van en línea con una investigación anterior en Frondel et al. (2008). 
También con el foco puesto en el comportamiento de los conductores de vehículos turismo, Safarzyńska y van den Bergh (2018) distinguen entre los comportamientos de los conductores que siguen prácticas rutinarias de los que denominan racionales. Estos últimos son proclives a ajustar sus planes de viaje óptimamente en respuesta a cambios en el precio de los combustibles y en la eficiencia de vehículos. Safarzyńska y van den Bergh (2018) encuentran que tanto los primeros como los conductores con aversión al riesgo son los que más contribuyen al efecto rebote. Al margen de sus hallazgos, la principal novedad de esta investigación radica en el abordaje de la cuestión de estudio que pone el énfasis en los aspectos sicológicos del comportamiento. Así, concluyen que las estimaciones sobre el uso del transporte en vehículos tienden a ignorar la racionalidad limitada de los conductores. Aunque para un ámbito que analizaremos más abajo, el trabajo de Safarzyńska y van den Bergh (2018) va en la línea del de Hediger et al. (2018), que enlazaron el análisis econométrico con la emergente "behavioral economics" investigando cómo los hogares respondían a mejoras en los sistemas de calefacción.

El análisis del ER en el sector del transporte también ha permitido ensanchar su análisis en el original artículo de Galvin (2016). En este caso la autora se aparta de la reacción esperada del aumento del tamaño del coche o de la distancia recorrida ante una mejora de la eficiencia para analizar los cambios en la aceleración y velocidad de los vehículos como reacción a esta mejora. De esta forma el ER sería consecuencia de cambios en los patrones de conducción de los usuarios. Como principal resultado se obtiene que la literatura disponible infraestima el efecto que la aceleración habitual de los vehículos puede provocar en la magnitud del ER tras una mejora de la eficiencia. Para el caso de los vehículos de combustión interna la infraestimación promedio es del $60 \%$, mientras que en los vehículos eléctricos se limita al 20,5\%.

Precisamente, la irrupción en el mercado de los vehículos eléctricos, híbridos y, en general, de consumo flexible, representa el último grupo de trabajos sobre el ER en el sector del transporte al que vamos a referirnos. JarkeNeuert y Perino (2020) dejan poca duda cuando afirman que el aumento de la eficiencia de los vehículos eléctricos provocará un aumento de las emisiones de $\mathrm{CO}_{2}$ en aquellos países en los que la generación de electricidad está sujeta a un esquema de comercio de derechos de emisión del tipo "cap and trade". Esto es precisamente lo que ocurre en el Régimen de comercio de derechos de emisión de la UE (Emissions Trading System -EU ETS-). Naturalmente, el aumento en las emisiones está anudado a la existencia de ER. Una nueva muestra del disenso en el hallazgo de evidencias científicas sobre el ER aparece cuando se compara este trabajo con el de Haan et al. (2006) para Suiza. Estos autores no encuentran evidencia de ER cuando se define en los habituales términos de comprarse un coche más grande o aumentar el promedio de los vehículos de los 
que se es propietario. No obstante, debe hacerse notar la escasa penetración de los vehículos eléctricos cuando se realizó esta investigación.

Pongamos ahora el foco en las estimaciones del ER en el sector industrial, no sin antes advertir que, después del sector servicios, el industrial es un sector marcadamente heterogéneo en el que la intensidad de demanda energética de las diferentes industrias varía significativamente. A pesar de ello abundan las investigaciones que lo abordan como un todo por lo que, en estos casos, los resultados deben tomarse con cautela. De hecho, el 79\% de los artículos analizados por Safarzadeh et al. (2020) abordan el sector en su totalidad. Dentro del conjunto del sector destacan las industrias en las que los procesos productivos requieren de la energía térmica en unos niveles que nos son compatibles con el origen eléctrico de la misma por lo que su procedencia deriva de la quema de combustibles fósiles en altos hornos. En este ámbito deben destacarse la industria del hierro y del acero junto con la del cemento. No muy lejos debe tenerse en cuenta a la industria del refino.

Una reciente visión panorámica del ER en la industria de once países miembros de la UE se debe a Cardoso et al. (2019), quienes descartan que la mejora de la eficiencia energética provoque un ER superior al $100 \%$. Cuando se alcanza o supera esta magnitud se utiliza el término "backfire effect" para referirnos al ER. Cansino et al. (2019) también descartan este efecto para el caso de España analizando el periodo 2000-2015.

Una visión exhaustiva de la relación entre el fomento de los programas de eficiencia energética en la industria y los problemas de ER a los que pueden dar origen se debe a Safarzadeh et al. (2020). Estos autores advierten de los dos lados por los que el ER puede aparecer. Efectivamente, el ER puede aparecer en el lado de la producción a través de un aumento en el uso de recursos en los procesos de fabricación, para lo que se emplearía parte de los ahorros derivados del menor gasto en consumo de energía (Smeets et al., 2014). Pero el ER también aparece por el lado de la demanda de los otros sectores productivos y de los sectores institucionales de la demanda final.

Tras rastrear 470 artículos extraídos de la Web of Science, el término "rebound effect" aparece como uno de los seis grupos de términos más citados por la literatura que analiza los programas de mejora de la eficiencia energética en la industria. El único país europeo que recibe un número elevado de artículos basados en sus casos de estudio es Suecia, compitiendo internacionalmente con China y EE.UU.

Limitándonos al caso europeo, de Safarzadeh et al. (2020) pueden extraerse los siguientes trabajos. Para las industrias intensivas en energía del Reino Unido Barker et al. (2007b) hallaron un ER del 19\% para el año 2007, mientras que Amjadi et al. (2018) estimaron un ER del 86\% para la industria pesada en Suecia. Por último, y para el conjunto de la UE, Smeets et al. (2014) 
encuentran un ER del $22 \%-30 \%$ asociado al reemplazo del uso de combustible de origen fósil por biocombustible.

A diferencia de los sectores del transporte e industrial, el resto de sectores productivos ha recibido una menor atención de los académicos. Probablemente su menor intensidad de demanda energética explique el menor interés. Aun así, la literatura ofrece hallazgos para sectores como la Agricultura y algunos subsectores del sector servicios como el de Educación.

Paul et al. (2019) analizaron el ER para en el sector agrícola alemán. A diferencia de los que ocurre en otros sectores productivos, en el caso de la agricultura el ER puede no derivarse del cambio en el comportamiento de los agentes económicos sino de causas naturales como la mayor resistencia de algunas plagas a los pesticidas. Estos autores detectan, aún sin cuantificar con precisión, los ERD y ERI como consecuencia en mejoras introducidas en las técnicas de irrigación. El resultado final es un aumento en el consumo de agua en los cultivos. Un análisis no muy alejado de la agricultura, pero desde una perspectiva más amplia que vincula el crecimiento económico con la desmaterialización, se encuentra en Petrides et al. (2018). Los autores conciben la desmaterialización como la reducción de la necesidad de uso de materiales y materias primas en los procesos productivos.

Finalmente, y en el caso de la Educación, Herring y Roy (2002) realizaron una temprana investigación sobre el ER en este subsector del sector servicios para el caso del Reino Unido. Sus resultados pusieron de manifiesto que la educación a distancia suponía un 90 \% menos de consumo de energía que la enseñanza universitaria presencial. Sin embargo, el uso de dispositivos electrónicos no reducía la huella de emisiones de $\mathrm{CO}_{2}$ cuando se compara con la enseñanza basada en material didáctico impreso en papel.

\subsection{Estimaciones del ER en edificios y hogares}

El sector servicios ha quedado fuera del subapartado anterior a pesar de ser un sector productivo determinante para el conjunto de la UE y de España, no sólo en términos de su aportación al PIB sino también de su consumo de energía. La razón de ello es que su principal demanda de energía -particularmente en el sector de comercio al por mayor, al por menor y en las administraciones públicas- está asociada al consumo realizado por los edificios que acogen estas actividades. De hecho, los edificios no residenciales representan la cuarta parte del parque total de edificios de la UE y son responsables de la tercera parte del consumo de energía del mismo (Grossman et al., 2016). De la misma manera y dejando a un lado el consumo de energía en el transporte, el sector de los hogares - que no es en este sentido considerado como productivo- también realiza su principal consumo de energía en sus viviendas. Por esa razón 
abordamos en este subapartado el ER en el funcionamiento de los edificios -para uso residencial y no residencial-y en el comportamiento de los hogares.

Hasta 2018, la UE daba muestras de estar en línea para alcanzar sus objetivos de eficiencia energética para 2020. De hecho, el consumo de energía en 2014 fue el más bajo desde la década de los 80 del siglo pasado e inferior también al requerido para cumplir el objetivo para 2020. Esta situación cambió a partir de 2019. Parte del camino para recuperar la senda hacia el logro de los objetivos mencionados pasa por mejorar la construcción de edificios y la eficiencia en la calefacción de espacios (Thomas y Rosenow, 2020); sobre esto último advirtieron tempranamente y para el caso de Irlanda Hull et al., (2009). En países como Dinamarca, sólo el consumo de energía para calefacción tendría asociado un ER del $31 \%$ (Andersen et al., 2020).

Para el conjunto de los edificios residenciales de la UE-28 y Noruega Galvin (2014) analizó el ER definiéndolo como la elasticidad de la demanda de energía a la eficiencia energética. Sin embargo, la misma autora reconocía que era una estimación imprecisa. Muestra de ello es que para los países con mayor antigüedad en su pertenencia a la UE el ER variaba en un rango del $0 \%$ al 50 $\%$ mientras que para los países de más reciente incorporación el rango oscilaba entre el 100\% y el 550\%. Seebauer (2018) para el caso de Austria analizó el ER en las viviendas de uso residencial después de realizar una inversión en la mejora del aislamiento. Los resultados que obtenían mostraban que los hogares con mayor renta son los más propensos al ER. El tamaño del ER se contrarresta con los valores pro ambientales y conciencia de consumo respetuoso con el medio ambiente de los inquilinos.

\section{El efecto rebote en las normativas europeas y españolas. Ausencias y propuestas}

Las medidas orientadas a mejorar la eficiencia energética han formado parte de la política energética de la UE desde los años 70 del siglo pasado (Bertoldi, 2020). Sin embargo, las primeras medidas estaban motivadas por las crisis del petróleo y la necesidad de reducir la dependencia exterior de este combustible, mientras que las medidas más recientes están motivadas por los intentos de frenar el calentamiento global.

Como es sabido, la UE actúa como alta parte contratante en los tratados internacionales relacionados con cuestiones medioambientales y, también, con la lucha contra el Cambio Climático. En este sentido el documento remitido por la UE a la ONU para fijar sus compromisos (la denominada "nationally determined contribution -NDC-") estableció el compromiso de reducir para el año 2030 el nivel de emisiones de GEI en un $40 \%$ con respecto al 
volumen de emisiones del año 1990. De ese compromiso y de otros anteriores se derivan las acciones orientadas a promover la eficiencia energética por cuanto la mayor parte de las emisiones proceden del uso de la energía. A este compromiso obligatorio se unía la intención de convertir a la economía de la EU en una economía con cero emisiones netas de GEI. Esto implica no que las actividades económicas realizadas en la UE estén para ese año libres de emisiones de GEI, sino que las actuaciones compensatorias de las mismas (por ejemplo, la existencia de superficies forestales que actúen como sumideros o la implantación de tecnologías de secuestro de estas emisiones) serán suficientes para compensar el volumen total de emisiones. Este objetivo último es el corazón de la denominada European Green Deal (Comisión Europea, 2019), y está en línea con los mencionados compromisos adoptados en el Acuerdo de París (Naciones Unidas, 2015), respaldados luego por el Consejo Europeo en diciembre de 2019 (Consejo Europeo, 2019).

Para ubicar con mayor precisión el documento que determina la hoja de ruta hacia una economía con cero emisiones netas de GEI hay que ir a la Resolución del Parlamento Europeo de marzo de 2019, donde define esta estrategia de largo plazo (Parlamento Europeo, 2019), y a la Resolución del mismo de enero de 2020 sobre la mencionada European Green Deal (Parlamento Europeo, 2020). Las actuaciones orientadas a promover la mejora de la eficiencia energética están en línea con los documentos y compromisos antes citados. De hecho, la firma de los Acuerdos de París en 2015 y su ratificación el año siguiente explican la aprobación de la Directiva 2018/2002/UE que actualiza la anterior Directiva 2012/27/UE aumentando el objetivo de mejora en la eficiencia energética del $20 \%$ al $32,5 \%$. Estas directivas plasman el compromiso actual de la UE con la mejora de la eficiencia energética. A partir de las Directivas cada estado miembro tiene que desarrollar sus planes nacionales.

Para llegar al objetivo del 32,5\% debe recordarse el camino recorrido. En particular, el Consejo Europeo de 23 y 24 de octubre de 2014 apoyó en un primer momento el objetivo de eficiencia energética del $27 \%$ para 2030 a escala de la Unión, con miras a revisarlo en 2020 teniendo en mente un objetivo del nivel del 30\% para la Unión (finalmente fue el citado 32,5\%). En su resolución de 15 de diciembre de 2015 titulada «Hacia una Unión Europea de la Energía», el Parlamento Europeo hizo un llamamiento a la Comisión para que evaluara también la viabilidad de un objetivo de eficiencia energética del 40\% para el mismo horizonte temporal. Este objetivo no fue finalmente asumido, aunque la UE demostró un claro liderazgo internacional en sus compromisos que iba más allá de lo asumido en los Acuerdos de París de 2015.

España ha seguido el camino de ambición de la UE fijando objetivos nacionales que han superado los propios de la Unión. Los planes nacionales españoles han cubierto los periodos 2005-2007, 2008-2012, 2011-2020 (el último 
actualizado tras la aprobación de la Directiva 2012/27/EU para el periodo 20142020) (Ministerio de Industria Energía y Turismo, 2005, 2007, 2011 y Ministerio de Industria Energía y Turismo, 2014). Un análisis en profundidad de los planes de Acción de España se ofrece en Román-Collado y Sanz (2017).

En ninguno de los documentos legales mencionados -ni a nivel mundial, ni de la UE ni de España- se aborda de manera directa el problema del ER como barrera para alcanzar los objetivos de mejora de la eficiencia energética. De manera práctica el problema se salva estableciendo los objetivos obligatorios en términos de niveles absolutos máximos para el consumo de energía primaria y de energía final. Esta elección ha resultado una opción mucho más clara que fijar el objetivo de mejorar la eficiencia energética a partir del cociente especificado en la Sección 2 de este artículo.

Se detecta por tanto una situación paradójica, por cuanto el consenso de los expertos en torno a la existencia del problema del ER no se ha traducido en ninguna actuación política tangible de alto nivel (Vivanco et al., 2016).

Lo anterior no es obstáculo para que en aplicación de la metodología seguida en este artículo sea posible identificar las principales medidas que la literatura ofrece para bloquear y reducir el problema del ER. Estas medidas sí es habitual encontrarlas referidas a sectores productivos específicos.

Por ejemplo, siguiendo a Safarzadeh et al. (2020) las políticas más utilizadas en los programas de mejora de la eficiencia energética en el sector industrial han sido la incorporación de estándares de eficiencia y evaluación ex post de los niveles de eficiencia alcanzados respecto de los niveles de partida (Ni, 2013; Shi, 2014, 2015; Andersson et al., 2018; Price et al., 2010) y el uso de los certificados blancos comercializables con la participación de empresas de servicios energéticos (Bertoldi et al., 2020). En este tipo de programas el apoyo de la eficiencia energética a partir de fondos públicos se suele entregar en forma de pago directo a las empresas o a los usuarios finales que adquieren equipos para mejorar la eficiencia energética. Las políticas de poner precios dinámicos a la energía o impuestos son también habituales. Por ejemplo y para evitar el ER en los inquilinos de viviendas tras la introducción de mejoras en el aislamiento, Kulmer y Seebauer (2019) proponen gravar el consumo de combustible con un tipo impositivo del 43\%. Sin salir del sector residencial Seebauer (2018) recomienda ser selectivo a la hora de seleccionar a los beneficiarios de ayudas públicas limitándolos a los usuarios que se muestran menos propensos a incurrir en ER. Esta recomendación, sin embargo, produciría severos problemas de inequidad, pues en su investigación se encuentra que los hogares más propensos a incurrir en ER son los de menor renta, que, según su lógica, deberían quedar excluidos del apoyo público.

También en el sector de los hogares, pero ahora en relación con los electrodomésticos con etiquetado energético, Galarraga et al., (2016) 
encuentran para el caso español que la política más efectiva para mejorar la eficiencia energética sin incurrir en ER es el uso de impuestos que graven estos electrodomésticos; los más eficientes serían gravados más suavemente (incluso pueden combinarse con un sistema de subsidios) y los menos eficientes, más duramente.

\section{Conclusiones}

La Unión Europea ha asumido un liderazgo internacional claro en la lucha contra el calentamiento global. Este liderazgo se ha puesto de manifiesto al asumir compromisos obligatorios en la mitigación de emisiones de gases de efecto invernadero que sobrepasan los establecidos en los Acuerdos de París de 2015. Como parte de esa acción ambiciosa también ha asumido objetivos en la mejora de la eficiencia energética en términos de reducción en un 32,5 $\%$ de sus niveles absolutos de consumo de energía. España ha sido uno de los estados miembros de la UE que también ha asumido un fuerte compromiso en ambos objetivos mostrado a través de los sucesivos planes nacionales de ahorro energético.

Este artículo permite concluir que existe un amplio consenso en la literatura sobre el riesgo de que las mejoras en la eficiencia energética provoquen un efecto rebote en las decisiones de los agentes económicos, hasta el punto de reducir los posibles ahorros en el consumo energético e incluso anularlos. También se concluye que el consenso anterior convive con un claro disenso en las estimaciones globales y sectoriales del tamaño del ER. Esto ha impedido que se pongan en marcha medidas de política energética tangibles para afrontar esta limitación.

Un análisis en profundidad de la normativa y las actuaciones orientadas a mejorar la eficiencia energética tanto en la UE como en España permiten concluir que, como era esperado, hay una casi total ausencia de referencias al problema del efecto rebote y más aún a cómo superarlo. Esto no impide que el artículo incluya algunas de las medidas que la literatura especializada recomienda para mitigar el problema.

Nuestra recomendación es que si la UE quiere seguir ejerciendo de manera clara el liderazgo en la lucha internacional contra el calentamiento global y el cambio climático debe unir, a los esfuerzos ya desplegados, medidas específicamente orientadas a afrontar el problema del efecto rebote. 


\section{Agradecimientos}

El autor agradece la ayuda del Ministerio de Ciencia, Innovación y Universidades a través del proyecto RTI2018-096725-B-I00, de la Cátedra de Economía de la Energía y Medioambiente Universidad de Sevilla-REE, la ayuda del grupo de investigación SEJ-132 Teoría Económica y Economía Política, del Departamento de Análisis Económico y Economía Política de la Universidad de Sevilla y la ayuda de la Universidad Autónoma de Chile (Chile).

\section{Referencias bibliográficas:}

Adetutu, M.O., A. Glass, A.J., y Weyman-Jones, T. G. (2015). Economy-wide Estimates of Rebound Effects: Evidence from Panel Data, vol. 37, pp. $1-27$.

Adetutu, M. O., Glass, A. J., y Weyman-Jones, T. G. (2016). Economy-wide estimates of rebound effects: Evidence from panel data. The Energy Journal, 37(3).

Amjadi, G., Lundgren, T., y Persson, L. (2018). The rebound effect in Swedish heavy industry. Energy Economics, 71, 140-148.

Andersen, K. S., Wiese, C., Petrovic, S., y McKenna, R. (2020). Exploring the role of households' hurdle rates and demand elasticities in meeting Danish energy-savings target. Energy Policy, 146, 111785.

Andersson, E., Arfwidsson, O., y Thollander, P. (2018). Benchmarking energy performance of industrial small and medium-sized enterprises using an energy efficiency index: Results based on an energy audit policy program. Journal of cleaner production, 182, 883-895.

Arocena, P., Gómez, A., y Peña, S. (2016). La eficiencia energética, el efecto rebote y el crecimiento económico. Papeles de Energía, número 2, diciembre 2016.

Barker, T., Ekins, P., y Foxon, T. (2007 a). The macro-economic rebound effect and the UK economy. Energy Policy, 35(10), 4935-4946.

Barker, T., Ekins, P., y Foxon, T. (2007 b). Macroeconomic effects of efficiency policies for energy-intensive industries: the case of the UK Climate Change Agreements, 2000-2010. Energy Economics, 29(4), 760-778.

Bertoldi, P. (2020). Overview of the European Union policies to promote more sustainable behaviours in energy end-users. In Energy and Behaviour (pp. 451-477). Academic Press.

Bertoldi, P., Rezessy, S., Lees, E., Baudry, P., Jeandel, A., y Labanca, N. (2010). Energy supplier obligations and white certificate schemes: Comparative analysis of experiences in the European Union. Energy Policy, 38(3), 1455-1469. 
Cansino, J.M. 2020. El riesgo del efecto rebote en la descarbonización en Toral, P. Retos de la transición energética: España ante la descarbonización. Editorial jurídica SEPIN, Madrid. En prensa.

Cansino, J. M., Román-Collado, R., y Merchán, J. (2019). Do Spanish energy efficiency actions trigger JEVON'S paradox? Energy, 181, 760-770.

Comisión Europea. 2015. Comunicación de la Comisión al Parlamento Europeo, al Consejo, al Comité Económico y Social Europeo y al Comité de las regiones. Cerrar el círculo: Un plan de acción de la UE para la economía circular. COM/2015/0614 final. Disponible en https://eur-lex. europa.eu/legal-content/ES/TXT/?uri=CELEX:52015DC0614 Última consulta, 4 de septiembre de 2020.

Comisión Europea. 2019. A European Green Deal. Striving to be the first climateneutral continent https://ec.europa.eu/info/strategy/priorities-2019-2024/ european-green-deal_en Última consulta, 9 de septiembre de 2020.

Consejo Europeo, 2020. Conclusiones adoptadas por el Consejo Europeo en su reunión extraordinaria de los días 17, 18, 19, 20 y 21 de julio de 2020. https://www.consilium.europa.eu/media/45124/210720-euco-finalconclusions-es.pdf Última consulta, 8 de septiembre de 2020.

Consejo Europeo. 2019. European Council meeting (12 December 2019). Conclusions https://www.consilium.europa.eu/media/41768/12-eucofinal-conclusions-en.pdf

Craglia, M., y Cullen, J. (2020). Do vehicle efficiency improvements lead to energy savings? The rebound effect in Great Britain. Energy Economics, 104775.

De Haan, P., Peters, A., y Scholz, R. W. (2007). Reducing energy consumption in road transport through hybrid vehicles: investigation of rebound effects, and possible effects of tax rebates. Journal of Cleaner Production, 15(1112), 1076-1084.

Douglas, P.H. (1976). The Cobb-Douglas Production Function Once Again: Its History, Its Testing, and Some New 477 Empirical Values. Journal of Political Economy, 84(5), 903-15.

Duarte, R., Sánchez-Chóliz, J., y Sarasa, C. (2018). Consumer-side actions in a low-carbon economy: A dynamic CGE analysis for Spain. Energy Policy, $118,199-210$.

Fink, A. (2019). Conducting research literature reviews: From the internet to paper. Sage publications.

Frondel, M., Ritter, N., y Vance, C. (2012). Heterogeneity in the rebound effect: Further evidence for Germany. Energy Economics, 34(2), 461-467.

Frondel, M., Peters, J., \& Vance, C. (2008). Identifying the rebound: evidence from a German household panel. The Energy Journal, 29(4). 
Galarraga, I., Abadie, L. M., y Kallbekken, S. (2016). Designing incentive schemes for promoting energy-efficient appliances: a new methodology and a case study for Spain. Energy Policy, 90, 24-36.

Galvin, R. (2016). Rebound effects from speed and acceleration in electric and internal combustion engine cars: An empirical and conceptual investigation. Applied Energy, 172, 207-216.

Galvin, R. (2014). Estimating broad-brush rebound effects for household energy consumption in the EU 28 countries and Norway: some policy implications of Odyssee data. Energy Policy, 73, 323-332.

Greening, L. A., Greene, D. L., y Difiglio, C. (2000). Energy efficiency and consumption - the rebound effect - a survey. Energy policy, 28(6-7), 389401.

Grossmann, D., Galvin, R., Weiss, J., Madlener, R., y Hirschl, B. (2016). A methodology for estimating rebound effects in non-residential public service buildings: Case study of four buildings in Germany. Energy and Buildings, 111, 455-467.

Guerra, A. I., y Sancho, F. (2011). An analysis of primary energy requirements and emission levels using the structural decomposition approach: The Spanish case. En María Llop (ed) Air Pollution Economic Modelling and Control Policies (pp. 112-131).

Gillingham, K., Rapson, D., y Wagner, G. (2016). The rebound effect and energy efficiency policy. Review of Environmental Economics and Policy, 10(1), 68-88.

Hediger, C., Farsi, M., y Weber, S. (2018). Turn it up and open the window: On the rebound effects in residential heating. Ecological Economics, 149, 21-39.

Herring, H. (2004). Rebound Effect of Energy Conservation. Encyclopedia of Energy, 237-244.

Howarth, R.B. (1997). Energy Efficiency and Economic Growth. Contemp. Economic Policy, 15(4), 1-9.

Hull, D., Gallachóir, B. P. Ó., y Walker, N. (2009). Development of a modelling framework in response to new European energy-efficiency regulatory obligations: the Irish experience. Energy Policy, 37(12), 5363-5375.

International Energy Agency. 2020. Tracking Clean Energy Progress report. Disponible en https://www.iea.org/topics/tracking-clean-energy-progress Última consulta, 5 de agosto de 2020.

Jarke-Neuert, J., y Perino, G. (2020). Energy efficiency promotion backfires under cap-and-trade. Resource and Energy Economics, 62, 101189.

Kulmer, V., y Seebauer, S. (2019). How robust are estimates of the rebound effect of energy efficiency improvements? A sensitivity analysis of consumer heterogeneity and elasticities. Energy Policy, 132, 1-14. 
Lin, B., y Liu, X. 2012. Dilemma between economic development and energy conservation: Energy rebound effect in China. Energy, 45(1), 867-873.

Lin, B., Chen, Y., y Zhang, G. 2017. Technological progress and rebound effect in China's nonferrous metals industry: An empirical study. Energy Policy, 109, 520-529.

Liobikienè, G., \& Butkus, M. (2017). The European Union possibilities to achieve targets of Europe 2020 and Paris agreement climate policy. Renewable Energy, 106, 298-309.

Matos, F. J., y Silva, F. J. (2011). The rebound effect on road freight transport: Empirical evidence from Portugal. Energy Policy, 39(5), 2833-2841.

Ministerio de Industria Energía y Turismo. 2014. Plan Nacional de Acción de Eficiencia Energética 2017-2020 Energy Saving and Efficiency Action Plan 2014-2020. Aprobado por acuerdo del Consejo de Ministros en Abril de 2014.

Ministerio de Industria, Turismo y Comercio. 2005. Plan de Acción 20052007: Estrategia de Ahorro y Eficiencia Energética en España 2004-2012. Aprobado por acuerdo del Consejo de Ministros del 6 de julio de 2005.

Ministerio de Industria, Turismo y Comercio. 2007. Plan de Acción 2008-2012: Estrategia de Ahorro y Eficiencia Energética en España 2004-2012 (E4). Aprobado por acuerdo del Consejo de Ministros el 20 de julio de 2007.

Ministerio de Industria, Turismo y Comercio. 2011. PLAN NACIONAL DE ACCIÓN DE EFICIENCIA ENERGÉTICA 2017-2020. Aprobado por acuerdo del Consejo de Ministros el 29 de julio de 2011.

Naciones Unidas. 2015. Acuerdo de París. https://unfccc.int/files/essential background/convention/application/pdf/spanish paris agreement.pdf Úlima consulta, 9 de Septiembre de 2020.

$\mathrm{Ni}, \mathrm{C}$. C. (2013). Potential energy savings and reduction of $\mathrm{CO}_{2}$ emissions through higher efficiency standards for polyphase electric motors in Japan. Energy policy, 52, 737-747.

Ordóñez, M., Arcos, Á., Cansino, J. M., y Román-Collado, R. (2019). Efectos económicos y medioambientales de la electro-movilidad en España. Un análisis input output medioambientalmente. Economía industrial, 411, 8597.

Parlamento Europeo. 2019. European Parliament resolution of 14 March 2019 on climate change - a European strategic long-term vision for a prosperous, modern, competitive and climate neutral economy in accordance with the Paris Agreement (2019/2582(RSP)) https://oeil.secure.europarl.europa. eu/oeil/popups/ficheprocedure.do?lang=en\&reference=2019/2582(RSP). Última consulta, 9 de septiembre de 2020. 
Parlamento Europeo. 2020. European Parliament resolution of 15 January 2020 on the European Green Deal (2019/2956(RSP)) https://oeil.secure. europarl.europa.eu/oeil/popups/ficheprocedure.do?lang=en\&reference $=2$ 019/2956(RSP). Última consulta, 9 de septiembre de 2020.

Paul, C., Techen, A. K., Robinson, J. S., y Helming, K. (2019). Rebound effects in agricultural land and soil management: Review and analytical framework. Journal of cleaner production, 227, 1054-1067.

Petrides, D., Papacharalampopoulos, A., Stavropoulos, P., y Chryssolouris, G. (2018). Dematerialization and Environmental Sustainability: Challenges and Rebound Effects. Procedia CIRP, 72, 845-849.

Price, L., Wang, X., y Yun, J. (2010). The challenge of reducing energy consumption of the Top-1000 largest industrial enterprises in China. Energy Policy, 38(11), 6485-6498.

Román-Collado, R., y Sanz, M. 2017. Analysis of energy end-use efficiency policy in Spain. Energy Policy 101, 436-446.

Safarzadeh, S., Rasti-Barzoki, M., y Hejazi, S. R. (2020). A review of optimal energy policy instruments on industrial energy efficiency programs, rebound effects, and government policies. Energy Policy, 139, 111342.

Safarzyńska, K., y van den Bergh, J. C. (2018). A higher rebound effect under bounded rationality: Interactions between car mobility and electricity generation. Energy Economics, 74, 179-196.

Seebauer, S. (2018). The psychology of rebound effects: explaining energy efficiency rebound behaviours with electric vehicles and building insulation in Austria. Energy research \& social science, 46, 311-320.

Shi, X. (2015). Application of best practice for setting minimum energy efficiency standards in technically disadvantaged countries: Case study of Air Conditioners in Brunei Darussalam. Applied Energy, 157, 1-12.

Shi, X. (2014). Setting effective mandatory energy efficiency standards and labelling regulations: A review of best practices in the Asia Pacific region. Applied Energy, 133, 135-143.

Smeets, E., Tabeau, A., van Berkum, S., Moorad, J., van Meijl, H., y Woltjer, G. (2014). The impact of the rebound effect of the use of first generation biofuels in the EU on greenhouse gas emissions: A critical review. Renewable and Sustainable Energy Reviews, 38, 393-403.

Thomas, S., y Rosenow, J. (2020). Drivers of increasing energy consumption in Europe and policy implications. Energy Policy, 137, 111108.

Tranfield, D., Denyer, D., y Smart, P. (2003). Towards a methodology for developing evidence-informed management knowledge by means of systematic review. British journal of management, 14(3), 207-222. 
Vélez-Henao, J. A., Vivanco, D. F., y Hernández-Riveros, J. A. (2019). Technological change and the rebound effect in the STIRPAT model: A critical view. Energy Policy, 129, 1372-1381.

Vivanco, D. F., Kemp, R., y van der Voet, E. (2016). How to deal with the rebound effect? A policy-oriented approach. Energy Policy, 94, 114-125.

Weale, G. 2020. EU 2030 Emissions target - a reality check. Documento del Energy Economics Centre for Environment Management, Resources and Energy (CURE). Ruhr University Bochum, Alemania. Disponible en https://www. wiwi.ruhr-uni-bochum.de/mam/content/cure/eu 2030 emissions target a reality check 14.07.2020.pdf. Última consulta, 4 de septiembre de 2020 .

Wei, T., y Liu, Y. (2017). Estimation of global rebound effect caused by energy efficiency improvement. Energy Economics, 66, 27-34. 
